\title{
Envejecimiento e imaginario social en la ciudad de Trujillo
}

\author{
Aging and imaginary social in the city of Trujillo \\ Casanova Hidalgo, Doris; Gonzáles Sánchez, María; Jara Vera, Rossemary; Márquez Vásquez, Elsa; \\ Miranda Troncoso, Adriana (*) y Gutiérrez Vásquez, Ramiro (**)
}

\section{RESUMEN}

Esta investigación tuvo el propósito de identificar y describir los imaginarios sociales sobre el envejecimiento que presentó un grupo de personas adultas mayores de ambos sexos de la ciudad de Trujillo. La metodología empleada fue de enfoque cualitativo en la que se aplicó, mediante una entrevista, un cuestionario de frases incompletas de forma aleatoria. Se trabajó con una muestra de 50 adultos mayores cuyas edades oscilaron entre 55 y 87 años de edad; 22 fueron varones y 28 fueron mujeres. Se concluyó que los varones resaltan sus experiencias que han acumulado, buenas y malas; se consideran personas sabias con derecho a opinar o a juzgar; así mismo, a aportar a las nuevas generaciones. Las mujeres se inclinan más por el lado emocional, tranquilas, en su mayoría viven sin preocupaciones. En ambos sexos se reconoce la pérdida de facultades físicas y mentales, la aparición de diferentes enfermedades, dolencias, falta de productividad. En los varones, en un mínimo porcentaje, existe preocupación por el aspecto sexual. También resaltan que lo más lindo de envejecer es contar con una familia, compartir con amigos, recordar experiencias positivas y tener la satisfacción de haber cumplido todas sus metas.

Palabras clave: Vejez, imaginario social, autopercepción, tercera edad.

\section{SUMMARY}

This research was aimed to identify and describe the imaginary social on aging presents a group of older adults of both sexes in the city of Trujillo. The methodology was qualitative approach that was applied by interview, questionnaire incomplete sentences randomly. We worked with a sample of 50 older adults whose ages ranged between 55 and 87 years old; 22 were men and 28 were women. It was concluded that highlight men who have accumulated their experiences, good and bad; they are considered wise persons entitled to review or judging; likewise, to contribute to new generations. Women are more inclined to the, quiet, emotional side mostly carefree living. In both sexes the loss of physical and mental faculties, the occurrence of various diseases, ailments, lack of productivity is recognized. In men, in a small percentage, there is concern about the sexual aspect. They also emphasize that the most beautiful of aging is to have a family, share with friends, remember positive experiences and have the satisfaction of having fulfilled all its goals.

Keywords: Old age, imaginary social, self-perception, seniors.

(*) Estudiantes del Programa de Maestría en Psicología Clínica de la Facultad de Psicología de la Universidad Peruana Cayetano Heredia.

(**) Docente Principal de la Facultad de Psicología de la Universidad Peruana Cayetano Heredia. 


\section{INTRODUCCIÓN}

Este es un estudio que se basó en el trabajo de Ruiz, Scipioni y Lentini (2008). Se replicó, sobre todo, la parte metodológica: el cuestionario, el procedimiento de obtención de información, la estructura de la presentación y comentarios de los resultados.

Al respecto de la variable vejez se sostiene que el envejecimiento de la población observable en Latinoamérica y el Caribe, desde la segunda mitad del siglo XX (Villa y Rivadeneira, 1999; citado en Viveros (2001) es parte del proceso de cambio demográfico global, cuyas transformaciones derivadas implican cambios cualitativos en las modalidades en que se organizan las sociedades, al mismo tiempo que sirven de marco a los cambios culturales y económicos que han acompañado a este proceso.

Según Viveros (2001) los indicadores de envejecimiento de la población muestran una gran heterogeneidad entre los países, consecuencia de sus disímiles tendencias demográficas. En 1995 la proporción de personas de 60 y más años en Guatemala, de transición demográfica moderada, apenas supera el $5 \%$, y Uruguay, con una transición avanzada y precoz, alcanza a $17 \%$. Salvo el caso uruguayo, y en menor medida el de Argentina y Cuba, la región está lejos de la situación de los países desarrollados. El crecimiento de la población adulta mayor es mucho más intenso que en los otros grupos etarios y las proyecciones alertan sobre un vertiginoso envejecimiento en la primera mitad del siglo XXI.

Desde la óptica de CEPAL. CELADE (2004) en este nuevo siglo, además del crecimiento de la población, hay otros temas demográficos que están adquiriendo importancia política, económica y social. El envejecimiento de la población es uno de ellos, por sus fuertes repercusiones en el desarrollo de los países y por la amplia variedad de sectores a los que afecta (salud, educación, infraestructura y comercio, entre otros).

América Latina y el Caribe (CEPAL: CELADE (2004) por el avance de la transición demográfica, está envejeciendo paulatina pero inexorablemente. Se trata de un proceso generalizado; aunque con niveles variables, todos los países de la región marchan hacia sociedades más envejecidas. Dos características de este proceso motivan una preocupación urgente. En primer lugar, el envejecimiento se produce y se seguirá produciendo en el futuro a un ritmo más rápido que el registrado históricamente en los países hoy desarrollados. En segundo lugar, se dará en un contexto caracterizado por una alta incidencia de pobreza, una persistente y aguda inequidad social, un escaso desarrollo institucional, una baja cobertura de la seguridad social y una probable tendencia hacia la disminución del tamaño de las fuentes de apoyo producto de los cambios en la estructura y composición familiar. En este contexto, el envejecimiento es uno de los retos demográficos más importantes que enfrentarán los países de la región en el siglo XXI. El desafío consiste en hacer frente a este fenómeno mediante la definición de estrategias claras que garanticen, junto con la sostenibilidad de las finanzas públicas y la capacidad de cumplir con objetivos fundamentales de política presupuestaria, un nivel de vida digno para las personas mayores, que les permita verse favorecidas por el bienestar económico de su país y participar activamente en la vida pública, social y cultural (CEPAL. CELADE, 2004).

\section{Envejecimiento.}

Desde antaño se trata de una problemática poco abordada, en la medida que era muy poca la cantidad de población que estaba en esta etapa. Pero, según Martínez, Polo y Carrasco (2002) los progresos de la industrialización condujeron a una disolución cada vez más acentuada de la célula familiar, que unido al considerable y progresivo envejecimiento de la población obligaron a la sociedad a crear leyes, normativas y programas que aborden y den solución a los problemas que plantea la vejez de su población. Es una de las preocupaciones más urgentes de los Estados en el momento actual.

Estos cambios, sostienen Martínez, M., Polo, M. y Carrasco, B. (2002), en las formas de vida acentuaron la marginación social del anciano, y él mismo suele sentirse con frecuencia superviviente de un mundo que le es cada día más ajeno, hasta el punto que el tiempo que el anciano considera el suyo está en el pasado ("en mis tiempos...") porque la época que vive pertenece a los jóvenes. La jubilación que la sociedad impone al hombre de cierta edad, que se encuentra en una buena situación física e intelectual supone su apartamiento social definitivo y la perdida de su lugar en la sociedad que lo margina y en la que los valores propios de la juventud devalúan los valores que el anciano puede ofrecer al grupo humano al que pertenece. La experiencia acumulada por los años no tiene gran valor en la sociedad tecnocrática actual, donde prima la competitividad y es necesaria una 
rápida adaptación a las nuevas tecnologías que se renuevan constantemente.

En la actualidad hay mayor interés por estudiar y comprender esta etapa de la vida. Así, en concordancia con González (2007) al referirse al envejecimiento es necesario mencionar que existen dos procesos, el de envejecimiento individual y el de envejecimiento de la población. En el envejecimiento individual se debe tener en cuenta dos grandes factores: los endógenos (salud, genética, personalidad, entre otros) y los exógenos (estrés, tabaquismo, alimentación, higiene, medio ambiente, entre otros). En cambio, para la misma autora, el envejecimiento de la población de un país se encuentra directamente ligado a su desarrollo socioeconómico, tales como: a) Reducción de la tasa de natalidad; b) Aumento de la esperanza de vida y c) Movimientos migratorios de la población.

Según González (2007) el envejecimiento individual, es la toma de conciencia de cómo cada uno percibe su edad, es personal. Sostiene que el cuándo nos sentimos viejos puede ser en un momento dado y no necesariamente en un aniversario, o puede darse volverse consciente con alguna expresión que oímos en los jóvenes o niños al referirse a nuestra persona. Agrega que el envejecimiento es un proceso de deterioro fisiológico, progresivo, irreversible, bien definido e independiente de episodios patológicos que marcan la vida de un individuo y nos acerca cada día más a la muerte. González (2007) dice que el rechazo a envejecer, tal vez se deba, al percibir un cierto impedimento que se tiene de que ya no se podrán realizarse ciertas actividades. Agrega, pero, esta percepción aparece en todas las etapas del crecimiento: Por ejemplo, cuando somos niños queremos ser adultos para realizar ciertas cosas, y cuando somos adolescentes deseamos ser niños o pasar rápidamente a la edad adulta.

Para esta autora, lo importante de esta etapa adulta mayor, como de cualquier otra, es vivirla plenamente, pues algo triste que puede ocurrirle a un ser humano es privarse de muchas cosas en el transcurso de los años para realizarlas en la vejez, y una vez que ésta llega, lamentarse de todas las cosas de las que se privó en su juventud. Un arrepentimiento poco constructivo es el que se presenta por las cosas o situaciones que no se hicieron o vivieron, porque por lo menos en las otras, nos dimos la oportunidad de experimentar (González, 2007). Los síntomas del envejecimiento se presentan poco a poco; por ejemplo, cuando nos damos cuenta que nos cuesta levantarnos, que la lectura nos cansa más rápido. Cuando nos damos cuenta que ya no podemos ver bien, u oir como antes. El cansancio rápido ante ciertos ejercicios, aparición de algunos dolores de las articulaciones, de la espalda, de las piernas; entonces, conscientes o no, nuestro cuerpo ha envejecido.

Pero, la edad se manifiesta no solo en nuestro cuerpo, sino también en el cambio de decisiones, gustos y actitudes mentales (González, 2007). La manera en que la persona se adapta al envejecimiento y las circunstancias que este implica, como son las enfermedades, utilización de prótesis, bastones, múltiples medicinas, entre otros (auxiliares todos para el bienestar), serán las condiciones para distinguir, lo que ella llama, el envejecimiento fisiológico del patológico. Así, el estado tanto físico como psíquico dependerá, en gran parte, de las medidas preventivas que se hayan tomado en el transcurso de la vida. Como en cualquier proceso, el envejecer se ve sometido a las actitudes personales, tanto negativas como positivas, y dependiendo de cuál sea nuestra convicción, se percibirá e interpretará el envejecer como una enfermedad, o en su defecto como un proceso que día a día nos impone una serie de adaptaciones y cambios de actitud (González, 2007).

Adoptar una postura negativa sobre esta edad mayor, se puede acentuar más por medio del mensaje social (donde hay un gran culto por la juventud), lo que finalmente llevará a una disminución de la estima personal. Nuestro yo deja de valorarse como antes porque el cuerpo envía una imagen muy diferente de la que se tenía o se tiene de uno mismo (González, 2007).

\section{Vejez}

En la línea de González (2007) la vejez se puede abordar desde cuatro puntos de vista: 1) Cronológica, 2) Físico-biológica, 3) Psicoafectiva y 4) Social. La primera se relaciona con la edad, que en nuestra sociedad es normalmente a la edad de 60 a 65 años que se marca la vejez; edad que en cierta medida coincide con la de la jubilación. Así, la edad cronológica solo servirá para marcar una realidad bien objetiva.

Con respecto al envejecimiento físico, González (2007) dice que hay diferentes teorías y, aunque los cambios son de naturaleza biológica, también provocan cambios en el ámbito psicológico. La autora sostiene que los cambios físicos (salvo excepciones) se llevan a cabo paulatinamente, siendo los deportistas 
los que normalmente se dan más cuenta de estos; en cambio, la mayoría de personas que no son deportistas, perciben esta situación cuando ven y sienten que estos cambios afectan sus actividades cotidianas. La imagen de sí mismas pueden cambiar en las personas que perciben las canas, las arrugas y el desecamiento de la piel. Sin embargo, observa la autora, que no todos los individuos envejecen al mismo ritmo y de la misma manera: hay muchas personas de más edad que están en mejores condiciones de salud que personas más jóvenes, González (2007), le llama envejecimiento diferencial, ya que no todos los cambios de la edad madura y de la vejez son tan nefastos, y que la vejez dependerá también de la calidad de la personalidad y de la actitud personal para enfrentar esta etapa de la vida (González, 2007).

En relación al envejecimiento psicoafectivo, González (2007) sostienen que las personas que han preferido sentar sus valores en la belleza física y en la apariencia corporal, es muy posible que tengan más problemas para asumir su vejez; en cambio, para aquellas personas que no pensaron en ésta preferencia como su meta de vida, el proceso del envejecimiento, además de anhelado, se desarrollará en las mejores condiciones, dada su actitud positiva para envejecer.

Referente a la edad social, González (2007), refiere que esta designa los papeles a desarrollar en la sociedad, y muchas veces hay un conflicto entre las diferentes edades. Por ejemplo, el que desea jubilarse antes porque no quiere a su trabajo, y el que desea jubilarse después debido a que su trabajo le da un sentimiento de identidad importante o una remuneración que no tendrá al jubilarse.

\section{Imaginario Social}

En relación al imaginario social, Ruiz, Scipioni, y Lentini (2008) sostienen que se trata de una constitución subjetiva, pero que aun cuando tienen mucha relación con la lógica, éstas se enlazan con las interacciones sociales y vivenciales que la cultura genera, está inmersa en el contexto de las prácticas sociales y los discursos de una época. Agregan que no existe entonces, una subjetividad que pueda aislarse de la cultura y la vida social, como tampoco una cultura que pueda aislarse de la subjetividad que la sostiene, entre ambas existe una mutua implicancia, una mutua producción. Al parecer, es importante no dejar de lado el encuentro de la historia con la historia del sujeto.
Lo psíquico y lo social, sostiene Castoriadis (1998), son radicalmente irreductibles lo uno a lo otro y al mismo tiempo, absolutamente indisolubles, lo uno es imposible sin lo otro. El hombre sólo existe en y por la sociedad, sociedad que además es siempre histórica. Esta, se mantiene unida porque establece una red de significaciones sociales, creadas por quienes la conforman, constituyendo todo lo instituido y aceptado por consenso, orientando y dirigiendo la vida de la sociedad y la de los sujetos que la constituyen.

Por otro lado, siguiendo la línea de Castoriadis (2006) el imaginario social puede comprenderse como que los sujetos y las cosas son creaciones sociales, tanto en la forma general, como particular y lo único que le pertenece al sujeto es su psique misma, que tampoco sería capaz de establecerse sin la violenta imposición que ejerce sobre ella el individuo como grupo, con predominio masculino.

Según (Allidière, 2005; citada en Ruiz, Scipioni, y Lentini, 2008) en este contexto, van surgiendo nuevas subjetividades, que puede conllevar carga de angustia y ansiedad. Es común escuchar malestar e incomodidad, efectos donde la imagen, lo instantáneo, el aislamiento, lo universal han borrado el valor de la palabra, las diferencias, las identidades. Se privilegia y se busca el cuerpo perfecto, y en la sociedad contemporánea la industria farmacéutica, las cirugías, las dietas, la buena imagen y pueden ayudar a prolongar la "juventud", borrando con ello las marcas del paso del tiempo. Además, Allidière, 2005; citada en Ruiz, Scipioni, y Lentini, (2008) sostiene, que en la vida cotidiana, estimulada por los medios masivos de comunicación, el ideal de eterna juventud puede llevar a la desaparición de las diferencias generacionales: la infancia se acorta, la adolescencia se alarga, la adultez aparece como incierta y se resiste; y la vejez se mantiene oculta y desmentida.

Entonces, es posible que junto a la imagen, la dimensión temporal también se vea cambiada. Así, el dinero que no logra comprar la juventud pero sí la imagen, puede lograr borrar las diferencias. El cuerpo del sujeto contemporáneo se convierte en mercancía y queda sometido así a la lógica del mercado. En este contexto posmoderno la persona que envejece, debe procesar la presencia en sí de un cuerpo cargado de significaciones sociales en una cultura que oferta la promesa de poder lograr alargar la vida sana a cambio de acatar las exigencias del libre mercado de consumo (Allidière, 2005; citada en Ruiz, Scipioni, y Lentini, 2008). 


\section{OBJETIVO}

Describir los imaginarios sociales que presenta un grupo de personas adultas mayores, de ambos sexos, en la ciudad de Trujillo.

\section{METODOLOGÍA}

La metodología empleada se orientó bajo un enfoque cualitativo. El instrumento aplicado para recolectar los datos, estuvo conformado por frases incompletas tomadas para tal fin de forma aleatoria. Se seleccionó y aplicó tres ítems con la finalidad de obtener información sobre las diferentes percepciones que manifiestan las personas entrevistadas. Los ítems fueron: 1) "ser vieja(o)para un varón/mujer es..."; 2) "lo más feo de envejecer para un varón/mujer es..." y 3) "lo más lindo de envejecer para un varón/mujer es...", estos ítems fueron tomados de Ruiz, Scipioni y Lentini (2008).

El instrumento de recolección de datos se aplicó a 50 adultos mayores de diferentes localidades pertenecientes a la ciudad de Trujillo, cuyas edades oscilaron entre 55 y 87 años de edad (22 varones y 28 mujeres). También se tomaron en cuenta las dimensiones: Pérdida de la salud, pérdida de la imagen, modificación en la sexualidad, pérdida del rol social y pérdida de proyectos, los mismos que fueron inferidos de la frase: "Lo más feo de ser viejo/ vieja es...". Asimismo, las dimensiones: Proyectos realizados, proyectos a realizar, experiencia y sabiduría, la descendencia, que fueron inferidos de la frase: "Lo más lindo de ser viejo/ vieja es...".

\section{ANÁLISIS DE DATOS}

Los resultados obtenidos han sido organizados y sintetizados aplicando la técnica de análisis de contenido. Luego se procedió a elaborar varias tablas a fin de presentar los datos cuantitativos, los mismos que permitieron contar con los insumos correspondientes para proceder a realizar la interpretación cualitativa de los resultados.

En la Tabla 1, se presenta los resultados de la primera frase: "Ser viejo para un varón/mujer es...". Se observa que seis varones y nueve mujeres la asocian mayormente a la "pérdida de la salud". En relación a la frase: "lo más feo de envejecer es...", quince varones y dieciocho mujeres la asocian, mayormente, con expresiones como: "no llegar a la meta sano y salvo"; "tener dolencias en el cuerpo"; "estar enfermos y no poder hacer nada"; "tener muchas enfermedades, no poder trabajar...".

El envejecimiento, ha sido motivo de preocupación desde siempre. El envejecimiento aparece a través de un conjunto de modificaciones morfológicas y fisiológicas que se dan como consecuencia de la acción del tiempo sobre las personas, lo que supone una disminución de la capacidad de adaptación de cada uno de los órganos, aparatos y sistemas, muy ligados a la salud.

Así, la teoría del desgaste de órganos y tejidos o teoría del ritmo de vida (BUENAS TAREAS (Acceso, enero de 2013) sostiene que las células del cuerpo se van estropeando conforme pasa el tiempo debido a su uso, algo similar a lo que ocurre con los componentes de una máquina. Lo que supone que las personas que han vivido forzando su cuerpo o que han tenido un estilo de vida poco saludable, viven menos. Esto explica que con el envejecimiento, comienza una serie de procesos de deterioro paulatino de órganos y sus funciones asociadas. Muchas enfermedades, como ciertos tipos de demencia, enfermedades articulares, cardíacas y algunos tipos de cáncer han sido asociados al proceso de envejecimiento.

Lo descrito líneas arriba y lo encontrado en esta investigación en relación a la frase: "ser vieja para un varón/mujer es...", muestra que el temor a la "pérdida de la salud" tiene mayor significancia en las mujeres que en los varones adultos mayores, Lo mismo, se puede afirmar en relación a la frase: "lo más feo de envejecer...", la mayoría de mujeres siguen asociando esta frase con la "pérdida de la salud", con menor frecuencia esta asociación se presenta en lo varones.

La Tabla 2 está mayormente relacionada con afirmaciones del tipo: "la persona se deforma físicamente", "muchas veces uno llega enfermo o mutilado", "tener arrugas", "estar inválido", lo que evidencia una clara relación con la "pérdida de imagen".

Tabla 1.

Ser vieja(o)...

Lo más feo de envejecer...

\begin{tabular}{ccccc} 
& Para un varón & Para una mujer & Para un varón & Para una mujer \\
\hline Pérdida de la salud & 6 & 9 & 15 & 18 \\
\hline
\end{tabular}


Tabla 2.

\begin{tabular}{ccccc}
\hline & \multicolumn{2}{c}{ Ser vieja(o)... } & \multicolumn{2}{c}{ Lo más feo de envejecer... } \\
& Para un varón & Para una mujer & Para un varón & Para una mujer \\
\hline Pérdida de la imagen & 2 & 7 & 8 & 6 \\
\hline
\end{tabular}

Tabla 3.

\begin{tabular}{ccccc}
\hline & \multicolumn{2}{c}{ Ser vieja(o)... } & \multicolumn{2}{c}{ Lo más feo de envejecer... } \\
& Para un varón & Para una mujer & Para un varón & Para una mujer \\
\hline Modificación en la sexualidad & 1 & - & 2 & - \\
\hline
\end{tabular}

En efecto, el proceso de envejecimiento da lugar a una mayor fragilidad orgánica, a un aumento de la vulnerabilidad frente a las enfermedades y en general a cualquier tipo de agresión externa. Además, el proceso de envejecer se caracteriza por ser la única edad que no introduce a otro ciclo de la vida y porque se puede percibir como la etapa más dramática de la vida: la etapa de "las pérdidas" y de "los temores". Pérdidas de todo tipo que se producen en esta etapa de la vida: del papel productivo, de la capacidad laboral, posibilidad de perder la pareja, los amigos, los hijos, disminución de eficiencia física y de la independencia psicológica, temor de enfermarse. Temor a la soledad, al aislamiento, a la incomprensión, a la falta de recursos económicos, a la discapacidad, a la fragilidad, a la dependencia. A la perdida de la imagen también se asocian los estereotipos, mayormente negativos para las personas de edad mayor. Así, Carbajo (2009) aludiendo a (Rodríguez Domínguez, 1989) sostiene que las interpretaciones negativas y peyorativas de la vejez, que son las más comunes, inciden en el deterioro de la persona mayor desde diversas perspectivas como la cronológica, la biológica o de salud, la psicológica o personal y la sociológica o comunitaria .

En la Tabla 3, se encuentra que los varones muestran más preocupación por su sexualidad a diferencia de las mujeres. Con un resultado de uno y dos para el varón, y sin ningún registro para la mujer, se han obtenido expresiones como: "no poder tener intimidad con la esposa".

La sexualidad es y ha sido una de las áreas del comportamiento humano más desconocida y en la que aún prima muchas veces la anécdota sobre el conocimiento científico. Y si esto es cierto a cualquier edad, lo es especialmente en personas de edad avanzada. La simple existencia de manifestaciones sexuales de cualquier tipo en los ancianos es sistemáticamente negada, rechazada o criticada por gran parte de la sociedad. En el trabajo de Herrera (2003; pp: 2) se puede observar sugerencias en torno a esta edad y la sexualidad. La autora menciona que: “... hace algunos años estamos asistiendo a un proceso de transición demográfica que obligadamente se debe acompañar de un proceso de revisión de la "cultura de la senilidad" donde se incluye también la variable de la sexualidad. Estamos, además, en la era del reciclaje: todo se recicla, incluso la vejez. Pero, en el área de la sexualidad está el peligro de "reciclar al viejo en un falso (e imposible) joven" con mitos y estereotipos sexuales basados en la prestación, la eficiencia y en la capacidad de identificarse con un joven con prodigiosas capacidades sexuales; concepto muy opuesto al antiguo que relacionaba la sexualidad con la procreación y consideraba al anciano como asexuado quedando excluido debido a la ineficiencia dada por la infertilidad. Pareciera que entre estos dos extremos, la sexualidad negada o la sexualidad impuesta no pudiera existir una imagen sexual en la que los componentes físicos, psicológicos y sociales se combinaran armoniosamente para crear una modalidad sexual específica que acompañe esta etapa de la vida".

Según Arias y Iacub (2013), se ha creído, hasta épocas recientes, de que la sexualidad no era posible ni deseable en la vejez. Pero, las investigaciones realizadas han mostrado que el declive de la sexualidad no está relacionado con la edad avanzada, sino, sobre todo, tiene que ver más con la viudez y el deterioro de la salud.

Los mismos autores sostienen que estudios longitudinales han mostrado que los adultos mayores, no solo son activos sexualmente, sino que también disfrutan de esta actividad (Ganen, 2002; Janus \& Janus, 1993; Master, Jonson \& Kolodny, 1995; citados en: Arias y Iacub, 2013). 
Los múltiples estereotipos relacionados con el erotismo en la vejez, según Iacub (2006; citado en Arias y Iacub, 2013) ) permite comprender sus significados en relación a la cultura y al entorno, en función del contexto de diversas concepciones y también en relación a mecanismos de poder que son los elementos que crean y mantienen estos estereotipos negativos. Sin embargo, desde una perspectiva positiva se debe de resaltar las posibilidades en lugar de las limitaciones, sobre la actividad sexual en la vejez, Iacub (citado en Arias y Iacub, 2013). Este último autor, propone centrar la problemática en el análisis del erotismo, al tratarse de una área más abarcadora que incluye el deseo, el amor e implica una amplia variedad de formas posibles de lograr placer.

En la tabla 4 se observa que la "pérdida del rol social", está muy asociada a la frase: "Ser viejo para un varón/mujer es...", con puntajes idénticos para ambos grupos. Con la frase: "lo más feo de envejecer es...", los puntaje son de siete para el varón y doce para la mujer. Aquí las respuestas más relevantes obtenidas han sido: "tener muchas enfermedades", no poder trabajar", "sentirse olvidado por sus hijos y familiares", "cuando dependemos de los demás (hijos, nietos)..."

Lo deseable es que la jubilación no sea sinónimo de deterioro y de pérdida económica, sino que se pueda gozar de un nuevo estilo de vida y de nueva libertad, disfrutar el tiempo que se tenga haciendo lo que se quiere y no pasarla de hospital en hospital. En esta etapa se teme envejecer, se teme el deterioro del cuerpo y la mente, se teme a las múltiples enfermedades, al sentimiento de inutilidad; e incluso, a las arrugas que se forman en nuestro rostro. En contra posición, es bueno decir: basta ya de miedos, envejecer no es el fin, es el inicio de una nueva etapa donde se pueda disfrutar al máximo. En tal sentido, es importante que antes que llegue esta edad, aprendamos cómo envejecer bien. La familia, pero sobre todo, los nietos se verán beneficiados por el rol que ocupan los abuelos en la familia luego de su jubilación (Alpízar, 2011).

Observando la Tabla 5, la frase: "ser viejo para el varón/mujer es...", registra una frecuencia de tres para el varón y cinco para la mujer. Asimismo, en la frase: "lo más feo de envejecer", las respuestas son tres y ocho; estas frases que están muy asociadas con la "pérdida de proyectos" y con expresiones como: "es cuando psicológicamente se siente viejo", "no tener energías de juventud", o "no poder hacer nada..."

Al parecer, llega un momento en la vida en que pensar en el futuro parece innecesario, llega un punto en que se ha alcanzado las metas o se puede creer que ya no hay más por hacer y lo único que queda es envejecer. Tal vez se sueñe con la jubilación y con todo ese tiempo que se puede disponer, pero al parecer esa es la única ilusión que queda al envejecer. Envejecer puede aterrar y por eso es posible evitar pensar en ese inevitable momento que llega cuando menos se espera. Pero así, se puede perder el horizonte de que es una parte de nuestra vida que también se debe planear, que no se debe esperar a que el envejecimiento llegue. Conforme se acerca la tercera edad, a menudo, se tiene la idea de que se está llegando al final. Llega la jubilación y con ésta, la sensación de que algo falta; no

Tabla 4.

\begin{tabular}{ccccc}
\hline & \multicolumn{2}{c}{ Ser vieja... } & \multicolumn{2}{c}{ Lo más feo de envejecer... } \\
& Para un varón & Para una mujer & Para un varón & Para una mujer \\
\hline Pérdida del rol social & 4 & 4 & 7 & 12
\end{tabular}

Tabla 5.

\begin{tabular}{ccccc}
\hline & \multicolumn{2}{c}{ Ser vieja... } & \multicolumn{2}{c}{ Lo más feo de envejecer... } \\
& Para un varón & Para una mujer & Para un varón & Para una mujer \\
\hline Pérdida de Proyectos & 3 & 5 & 3 & 8 \\
\hline
\end{tabular}

Tabla 6.

\begin{tabular}{ccccc}
\hline & \multicolumn{2}{c}{ Ser vieja... } & \multicolumn{2}{c}{ Lo más lindo de envejecer... } \\
& Para un varón & Para una mujer & Para un varón & Para una mujer \\
\hline Proyectos realizados & 3 & 9 & 8 & 18 \\
\hline
\end{tabular}


saber qué hacer con el tiempo del que se dispone y la necesidad de darle un nuevo sentido a la vida. Algunas opciones pueden ser pasar el tiempo con los nietos, salir de viaje o reunirse con viejas amistades. Puede ser que esto no sea suficiente, pero nunca es tarde para aprender y planear otras actividades.

Por otra parte, en la Tabla 6, las respuestas relacionadas a la frase: "lo más lindo de envejecer...", ante la frase: "ser viejo para un varón/mujer es...", tiene una puntuación de tres para los varones y nueve para las mujeres. Asimismo, para la frase: "lo más lindo de envejecer", los puntajes fueron ocho y dieciocho, respectivamente. Estas frases estuvieron muy asociadas a "proyectos realizados" y a expresiones como: "vivir agradecido de Dios por la vida", "tener una familia con quien dialogar", "ser libre del trabajo y dedicarse a viajar por el mundo..."

En la Tabla 7, se puede observar que existe la probabilidad de realizar proyectos o tener una continuidad de ellos, o finalmente puede ser visto como una amenaza de llegar a vieja(o) y no haber concluido los proyectos. Los resultados muestran que en la frase: "ser viejo para un varón/mujer es...", las mujeres obtienen un puntaje de dos y no se registra ningún puntaje en los varones. En cambio, en la frase: "lo más lindo de envejecer", se aprecia resultados similares para ambos géneros. Estos resultados podrían entenderse como un riesgo en la estabilidad emocional y podría afectar al proceso normal de envejecimiento.

Tal vez algunas de las mejores formas para ser y estar felices sea probar cosas nuevas, conocer personas, desarrollar nuevas habilidades y mantenerse ocupado física y mentalmente. No es necesario que sean cursos o talleres orientados exclusivamente a adultos mayores. Hay muchas actividades que es posible realizar, sólo es cuestión de encontrar la que mejor se adapte a los gustos y necesidades de cada persona. Incluso la simple adopción de un hobby o pasatiempo puede mejorar sustancialmente la calidad de vida. Entrar en la tercera edad no es sinónimo de decadencia. Al contrario, es una oportunidad de iniciar un nuevo ciclo. Al parecer, mantenerse activo y motivado es clave para estar bien física y emocionalmente. El tiempo del que se pueda disponer es una oportunidad de gozar la vida, renovarse y enriquecerse.

La Tabla 8 muestra que ante la frase: "ser viejo en el varón/mujer", surgen respuestas relacionadas a la "experiencia" y a la "sabiduría", así como también en la frase: "lo más lindo de envejecer". Para la primera frase, los varones obtienen una puntuación de trece y las mujeres de diez. Para la segunda frase, se halla doce para los varones y trece para las mujeres. Se tiene también que estas frases se relacionan con afirmaciones, tales como: "haber adquirido las buenas y malas experiencias", "la experiencia que tengo", "ser más sabias", "experiencia, prudencia", "experiencias agradables...".

Envejecer no es una debacle ni una edad dorada. Es una etapa tan rica y digna de ser vivida como las otras, es apasionante, con sus alegrías y sus dificultades. Da problemas, por supuesto, a todos los niveles: económicos, sociales, psicológicos, pero aún en estas condiciones debe abordarse estos problemas de frente y saber anticiparse a ellos. La psicoterapeuta María Hennezel afirma que envejecer es "un arte contradictorio: por una parte es un naufragio, por otra, una progresión" (Hennezel, 2009). Es un naufragio

Tabla 7.

\begin{tabular}{ccccc}
\hline & \multicolumn{2}{c}{ Ser vieja(o)... } & \multicolumn{2}{c}{ Lo más lindo de envejecer... } \\
& Para un varón & Para una mujer & Para un varón & Para una mujer \\
\hline Proyectos a realizar & - & 2 & 4 & 4 \\
\hline
\end{tabular}

Tabla 8 .

\begin{tabular}{lcccc}
\hline & \multicolumn{2}{c}{ Ser vieja... } & \multicolumn{2}{c}{ Lo más lindo de envejecer... } \\
& Para un varón & Para una mujer & Para un varón & Para una mujer \\
\hline Experiencia y sabiduría & 13 & 10 & 12 & 13 \\
\hline
\end{tabular}

Tabla 9.

\begin{tabular}{ccccc} 
& \multicolumn{2}{c}{ Ser vieja... } & \multicolumn{2}{c}{ Lo más lindo de envejecer... } \\
& Para un varón & Para una mujer & Para un varón & Para una mujer \\
\hline La descendencia & 1 & 2 & 6 & 6 \\
\hline
\end{tabular}


porque comporta numerosos duelos por la pérdida de la energía vital, la juventud perdida, la esperanza de vida, la salud (aparecen los achaques), familiares y amigos de la misma edad que fallecen, el trabajo y el éxito profesional, la autonomía personal, hasta la independencia económica. No obstante, en el peor de los casos, Hennezel (2009) sostiene que es posible experimentar un importante crecimiento interior y aprender a saborear la vida de otra forma, puesto que envejecer también es una ganancia en términos de experiencia, sabiduría, crecimiento interior y espiritual. El objetivo en la vejez no debe ser buscar el éxito y el reconocimiento exterior, sino que la satisfacción viene cuando se madura interiormente.

Finalmente, en la Tabla 9, se aprecia que la frase: "lo más lindo de envejecer" está muy ligado a la importancia que tiene la "descendencia". Se observa una puntuación similar para ambos géneros. En la frase: "ser viejo para el varón/mujer es...", se observa puntajes de uno para el varón y dos para la mujer. Estas frases se relacionan con: "la descendencia" y con expresiones como: "tener descendencia que aprenda a respetar a Dios y su prójimo", "estar rodeado de su esposa y familiares", "cuando los nietos e hijos se reúnen y nos brindan mucho amor", "ser abuelo...".

Suele suceder que cuando la pareja se ve en la etapa del nido vacío (cuando los hijos han dejado el hogar), consideran que ya no hay razón de existir como tal. Si los cónyuges no han sabido estrechar sus lazos afectuosos, de comunicación y respeto, puede ser un momento en que se puede desencadenar el rompimiento de los lazos de la pareja. Es por esto que es importante trabajar sobre la etapa de vejez, preparándose para no ser una carga económica, física o moral para la familia. Llegar a la vejez con calidad de vida, dependerá mucho del estilo de vida que se ha llevado en los años anteriores a la vejez. Si desde niños se educa y prepara para la vejez, la percepción que se tenga de este periodo de vida será diferente; quedaría fuera de contexto la marginación, la estigmatización y el poco respetado hacia las personas de estas edades. Mantener una actitud positiva ante la vida, permitirá estar preparado dignamente para la tercera edad, y no sólo para ella, sino también para la muerte (Nugent y Gómez. Acceso, 2013).

Los hallazgos de esta investigación se pueden sintetizar bajo el enfoque biopsicosocial como sigue: 1) A nivel biológico, la vejez se halla asociada a situaciones como pérdida de salud, dependencia física, debilidad física, es considerada como una etapa negativa; pero a lo largo de la vida, esta categoría es considerada como positiva. 2) A nivel psicológico, la vejez aparece asociada a situaciones como falta de vitalidad, de desesperanza, añoranza del pasado, lo cual es considerada como negativa. En cambio, la sabiduría, la experiencia, la satisfacción personal, la fortaleza de espíritu y la independencia se manifiestan como situaciones positivas. 3) A nivel social, la vejez se halla ligada a situaciones como la soledad y abandono, y la dependencia de otros, que son consideradas como negativas. En cambio, ser abuelos y tener una familia o red social de protección, se considera como lo positivo en esta etapa de la vejez.

\section{CONCLUSIONES}

1) Los varones encuestados revelan que "lo positivo de envejecer" son las experiencias que han acumulado, buenas y malas, se consideran personas sabias con derecho a opinar o a juzgar, así como también a aportar a las nuevas generaciones. Las mujeres se inclinan más por el lado emocional, en su mayoría, para ellas llegar a ser a ser viejas es estar tranquilas, vivir sin preocupaciones, sin embargo se presentan algunos casos especiales.

2) Para el ítem relacionado a "lo más feo de envejecer" ambos sexos hacen referencia a la pérdida de facultades físicas y mentales, así como también a la aparición de diferentes enfermedades, dolencias, falta de productividad. Específicamente, en el caso de los varones, en un mínimo porcentaje de ellos existe preocupación por el aspecto sexual, es decir, no poder satisfacer a su pareja.

3) Sobre el ítem: "Lo más lindo de envejecer es...", ambos sexos consideran el valor de la familia, el contar con su apoyo, compartir con amigos, recordar experiencias positivas $y$ tener la satisfacción de haber cumplido todas las metas de su vida.

\section{RECOMENDACIONES}

El conocimiento de estas representaciones sociales pueden ser un aporte para el conocimiento de la población adulta mayor de estas ciudades, y tiene un valor social en tanto que estos imaginarios sociales llevan a los miembros de la colectividad a realizar acciones congruentes con ellos, lo cual llevaría a proponer e implementar programas de intervención para fortalecer aquellas representaciones positivas y revertir las negativas en los mismos ancianos. 
Los hallazgos de esta investigación, puede ayudar a diseñar y llevar a cabo programas educativos a la población adulta, de jóvenes y niños con acciones preventivas respecto de aquellas situaciones que hacen sufrir a los adultos mayores, especialmente, en el núcleo familiar, ya que la persona adulta mayor necesita tener el apoyo de la familia y de la comunidad.

Los datos encontrados pueden servir de orientación y esclarecimiento para crear y fortalecer ambientes que brinden atención especializada para atender las necesidades médicas y psicosociales de las personas que se encuentran en esta etapa de la vida.

\section{REFERENCIAS BIBLIOGRÁFICAS.}

1. Ruiz, M., Scipioni, A. y Lentini, D. (2008). Vejez e imaginario social. Revista Electrónica de Psicología Política. En línea v.6 n.16 San Luis Mar. Universidad Nacional de San Luis.

2. Viveros, A. (2001). Envejecimiento y vejez en América Latina y el Caribe: Politicas públicas y las acciones de la sociedad. Proyecto Regional de Población CELADE-FNUAP (Fondo de Población de las Naciones Unidas). Centro Latinoamericano y Caribeño de Demografía (CELADE) - División de Población de la CEPAL. Área de Población y Desarrollo del CELADE. Santiago de Chile, diciembre.

3. CEPAL.CELADE (2004). Población, Envejecimiento y Desarrollo. Santiago de Chile.

4. Martínez, M., Polo, M. y Carrasco, B. (2002). Visión histórica del concepto de vejez desde la edad media. Revista de Enfermería y Humanidades Cultura de los Cuidados. Año VI, $\mathrm{N}^{\circ}$ 11. Departamento de Enfermería y Fisioterapia. Universidad de Alcalá. Madrid.
5. González, M. (2007). Los retos de la tercera edad. Nuevas oportunidades. México: Trillas.

6. Castoriadis, C.(1998). El ascenso de la insignificancia. Madrid: Cátedra.

7. Castoriadis, C. (2006). Figuras de lo pensable. México: FCE.

8. BUENAS TAREAS (Acceso, enero de 2013). Teoría del desgaste de órganos y tejidos. Accesible en: http://www.buenastareas.com/ensayos/ Teor\%C3\%ADa-Del-Envejecimiento-Desgaste-De$\% \mathrm{C} 3 \% 93$ rganos/49403259.html

9. Carbajo, M. (2009). Mitos y estereotipos sobre la vejez. Propuesta de una concepción realista y tolerante. Ensayos, Revista de la Facultad de Educación de Albacete, $\mathrm{N}^{\circ} 24$. Accesible en: http:// www.uclm.es/ab/educacion/ensayos

10. Herrera, A. (2003). Sexualidad en la vejez: Mito o realidad? Revista Chilena de Obstetricia y Ginecología; 68(2): 150-162. Santiago de Chile.

11. Arias, C. y Iacub, R. (2013). ¿Por qué investigar aspectos positivos en la vejez?. Contribuciones para un cambio de paradigma. Publ. UEPG Ci. Hum., Ling., Letras y Artes, Ponta Grossa, 21(2): 271-281, $\mathrm{jul} / \mathrm{dez} .12$.

12. Alpízar, I. (2011). Jubilación y calidad de vida. Revista de la Facultad de Ciencias Sociales de la Universidad Nacional de Costa Rica. Vol. 31, $\mathrm{N}^{\circ} .42,(15-26)$, Enero-Junio.

13.Nugent, J.y Gómez, V.(Acceso, 2013). Envejecimiento y expectativas de apoyo en la Vejez: Historias de dos generaciones de mujeres rurales costarricenses. Accesible en: http://ccp.ucr.ac.cr/seminario/pdf/ nugent.pdf 